Background Nottinghamshire Hospice's day therapy unit closed its doors to patients during the COVID-19 pandemic. Government advice was supporting discharge from hospital wherever possible (NHS England and NHS Improvement, 2020). When asked, more than four in five people say they would prefer to die at home (Hoare, Morris, Kelly, et al., 2015). Patients receiving care from specialist palliative care teams tend to do better than those without (Higginson \& Evans, 2010).

Aims To expedite the discharge of patients at end-of-life from hospital and prevent unnecessary hospital admission for those in the community so that more people achieve their preferred place of death (PPD) with the provision of a dedicated palliative care service.

Methods March-May 2020: consultations with fast track continuing care (FTCC), model planning, internal consultation, workforce transformation. May-July 2020: recruitment, service launch. July-March 2021: continuous service provision. We worked closely alongside FTCC to support patients either in hospital that wished to come home but needed a package of care or, patients that were deteriorating at home and required end-of-life care. An initial assessment by a registered nurse from the Hospice Outreach Discharge Support (HODS) team meant more timely referral into extended palliative community services including provision of specialist equipment and access to other hospice services e.g. bereavement support. It also focuses on the completion of EPaCCS and ReSPECT forms identifying PPD.

Results Number of patients treated by HODS May 20 March $21=195$.

$64 \%$ (124) died at home.

$23 \%$ (44) remained well enough to be discharged to domiciliary care agency.

$10 \%$ (20) admitted to hospital.

$3 \%$ (6) admitted to nursing home.

Average length of stay on HODS $=10$ days

PPD achieved $=95.87 \%[4]$

Conclusion The creation of HODS allows more people at end-of-life to remain in their PPD and with greater access to support services preventing carer burnout and unnecessary admission to hospital.

\section{P-126 HOSPICE@HOME TO PALLIATIVE RESPONSE TEAM}

Humaira Jamal, Elizabeth Kennedy. Peace Hospice, Watford, UK

10.1136/spcare-2021-Hospice. 143

\section{Background}

- Our Hospice at Home $(\mathrm{H} @ \mathrm{H})$ service supports 31 GP practices (population 315,000 patients).

- Pre- COVID-19 pandemic service provision of seven-days 9 am-5 pm was reviewed.

- Enhanced model developed supporting people wishing to die at home. (DIUPR local CCG 45.9 vs 46.6 England 2017 ONS).

- DIUPR increased during the pandemic.

\section{Aims}

- Increase ease of access to care provision at home through increased service hours.

- Increase medical support to develop a specialist palliative care virtual beds model.
- Uphold hospice's strategic aims and values of 'widening access' and 'reaching more people'.

- Uphold 'Ambitions for Palliative Care' - 'each person gets fair access to care' (National Palliative and End of Life Care Partnership, 2015).

Methods

- Collaborative appointment (Hospice and NHS) of new community palliative consultant.

- Use of triage priority rating.

- Agreed a rapid service pathway for those unstable/dying.

- New service launched September 2020 with increased hours ( 8 am to $10 \mathrm{pm}$ ) and ability to review patients same day.

- Supported out of hours by on-call palliative consultants.

- Virtual beds model supported by daily 'ward rounds' and updated plan of care.

- Data collection - number of referrals, referral source, diagnosis, place of death.

Results

\begin{tabular}{ll}
\hline & Nov 20-May 21 \\
\hline Referrals & 362 \\
Patients seen & 144 \\
Died & 166 \\
Discharged & 221 \\
Death Location\% & \\
Hospital & $4 \%$ \\
Patient's own home & $80 \%$ \\
Hospice & $11 \%$ \\
Care Home & $4 \%$ \\
\hline
\end{tabular}

Conclusion Service activity increased. $84 \%$ of patients died in their usual place of residence. Daily review of patient plans enabled continuity of care. Clear contact information required for patients and families as different service involvement can be confusing. Referral process between partner organisations needed re-discussion and review.

Future planning

- Priority ratings to be reviewed.

- Strengthen relationships with GP practices by attending Gold Standards Framework meetings.

- Increase use of data collection including user feedback to support the development of service to enable more patients to be reached in line with identified growing demand.

\section{P-127 A POPULATION HEALTH MANAGEMENT APPROACH TO END-OF-LIFE CARE: INCREASED PANDEMIC RESILIENCE}

Julie Barker. Nottingham and Nottinghamshire ICS, Nottingham, UK

10.1136/spcare-2021-Hospice. 144

Aims To meet the needs of the increasingly aged frail population (projected to almost double by 2050), an integrated population-based end-of-life care service, founded on Gold Standards Framework (GSF) principles and the Ambitions Framework is using a collaborative approach to learn lessons, address inequity and build on its solid foundations.

Methods Mid-Notts End of Life Care Together Alliance (EOLCT) spread GSF to GP practices and care homes, 
mainstreaming a proactive approach, later spread to the wider Notts Integrated Care System/ICS population. Local hospices are working in partnership with community, third sector and acute sector organisations to lead development and expand capacity and flexibility of the service. The partners led on advance care planning, Respect implementation, a new ICS end of life strategy, a co-designed care-co-ordination service and regular interdisciplinary training for all care providers. Progress in population-based end-of-life care was assessed using robust comparative audits of digital record-sharing (EPaCCS) data against whole-system GSF end-of-life care metrics.

Results Nottinghamshire data shows (trend 2019- 2021)

- Earlier patient identification (0.22-0.66\%).

- Earlier and increased advance care planning (ACP) discussions and improving quality Respect forms (audited).

- Those on register attaining preferred place of care/death (54.7-67\%).

- Reduced hospitalisation and system resource savings $(10 \%$ Quipp).

- Better outcomes for individuals (commendations, fewer complaints).

- Nottinghamshire's use of GSF and ACP laid the foundations for a later robust, generalist-led response to the COVID-19 pandemic.

Conclusion A key challenge for our health/social care systems is responding to the increasing care needs of frail co-morbid patients. Our strong foundations enabled quick integrated responses for the most vulnerable during the COVID-19 pandemic, toolkits for primary and social care at the onset and increased resilience and sustainability thereafter.

\section{P-128 THERAPEUTIC SERVICES MERGE INTO WELLBEING DEPARTMENT WITH ONE REFERRAL AND HOLISTIC FAMILY SUPPORT}

Chanelle Wilson, Lauren Draycott, Liana Gowen. Havens Hospices, Essex

\subsection{6/spcare-2021-Hospice. 145}

The traditional hospice setup has been developed with silos across therapeutic services - resulting in families receiving multiple referrals for support with little or no understanding of the wider influences and having to retell their stories. Combining Social work, Counselling, Physio, OT, Creative therapy, Complementary therapy, Family support and Spiritual care into a wellbeing department streamlines the process with one referral, one holistic assessment, one MDT and one familycentred therapeutic plan. Families access the right support at the right time.

Aims Early intervention; holistic family support; integrated services; robust referral pathways; preventing crisis.

Methods

- Internal audits.

- Review of current counselling demand.

- Review of best practice guidance.

- Embedding a social care model for social and wellbeing needs.

- Client feedback.

- Evaluations.

- Referral trends.
Results

- Reduction in counselling waiting lists.

- Improved early intervention to prevent crisis.

- Streamlined referral processes - one referral to wellbeing services.

- Improved multidisciplinary working.

- Strengthen relationships with stakeholders.

- Embedded holistic family assessments.

Conclusion Therapeutic services improve wellbeing and should be integrated. Families consistently tell professionals that they do not want to keep repeating their stories and communication is key. To provide person-centred care we must look at all the influencing factors and family dynamics and support play a key role. To improve family wellbeing, we must look at the whole picture. This way of working is innovative in hospices but best practice in all therapy and family-based settings. Further research in hospices could strengthen this practice.

\section{P-129 VOLUNTEER TELEPHONE SUPPORT SERVICE (VTSS) TO SUPPORT SELF-MANAGEMENT AND ACHIEVEMENT OF GOALS}

${ }^{1,2}$ Yolande Borthwick, 'Margaret Anne Garner. 'Strathcarron Hospice, Denny, UK; ${ }^{2}$ University of Glasgow, Glasgow, UK

\subsection{6/spcare-2021-Hospice. 146}

Aims To develop the role of the volunteer to provide telephone support for patient goals following a self-management approach.

Description It is recognised that continued self-management in a long-term condition is difficult to sustain. The lymphoedema specialist service has limitations for supporting longer term goals and providing the social support needs of some of the patient group. It was identified that positive regular input could assist in continued self-management. Volunteers were unable to continue their usual role in the clinic during the COVID-19 pandemic and it was acknowledged that their skills could support patients in a less illness focused method.

The Volunteer telephone support service (VTSS) was developed with input from both lymphoedema/lipoedema patients and volunteers. The documentation, established in collaboration with patients and volunteers, assists with background information, purpose of the call, a guide through the phone call and a reminder of any 'red flags' which would need urgent follow up. At present the VTSS runs one day fortnightly. Outcomes of the calls are documented and there is a de-brief for the volunteer with a specialist lymphoedema practitioner to highlight any issues or follow up required. The patients decide whether they wish for further contact and the timescales are mutually agreed with the volunteer. The documentation generated is scanned into the electronic notes as a record of the contact.

Evaluation So far (March - May) 27 patients have been supported by this service, 13 requested regular contact with the volunteers and nine appropriate review appointments generated.

Evaluation - by questionnaire to the service users as well as quotes obtained during phone contacts and feedback from the volunteers. The service has evaluated very positively by both the patients and the volunteers. 